\title{
PRIMER REGISTRO DE LA PRESENCIA Y REPRODUCCIÓN DEL ANURO Rhinella poeppigii (Tschudi, 1845) EN UN ÁREA ANTRÓPICA DEL DEPARTAMENTO DE LIMA, PERÚ
}

\author{
FIRST RECORD OF THE PRESENCE AND REPRODUCTION OF THE ANURAN \\ Rhinella poeppigii (Tschudi, 1845) IN AN ANTHROPIC AREA IN LIMA, PERU
}

\author{
Marta Williams León de Castro ${ }^{1}$ y Dennis Rey Sánchez ${ }^{2}$
}

\begin{abstract}
Resumen
Se reporta por primera vez para la desierto costero del departamento de Lima la especie anura Rhinella poeppigii, cuyo rango de distribución hasta la fecha son los bosques montanos de las estribaciones orientales de los Andes en Perú y Bolivia (Angulo et al., 2004). Además se describen sus eventos reproductivos, observados desde inicios de abril hasta fines de noviembre del 2012, en un estanque del Jardín Botánico Octavio Velarde Núñez de la Universidad Nacional Agraria La Molina. Durante la actividad reproductiva se registró el número de individuos participantes, su sexo, el sistema de apareamiento, y se describió y comparó el micro hábitat elegido para la reproducción. El sistema de riego en el área y los aumentos en la temperatura ambiental coincidieron en el inicio de los eventos reproductivos que fueron de tipo explosivo.

Palabras clave: Rhinella poeppigii, eventos reproductivos, apareamiento, jardín botánico.
\end{abstract}

\begin{abstract}
The presence of Rhinella poeppigii an anuran species that can be found in cloud forests of the Andean slopes and tropical moist forest in the Amazonian foothills of Peru and Bolivia (Angulo et al., 2004), is reported for the first time in the coastal desert of Peru, department of Lima. A description of its reproductive events is included, recorded from early April to late November 2012, in a small pond inside the "Octavio Velarde Núñez" Botanical Garden, at La Molina National Agrarian University. During the reproductive activity the number of individuals involved, their sex, and mating system were recorded as well as the microhabitat they chose for their reproduction. The irrigation system in the area and increases in environmental temperature triggered the onset of the explosive reproductive events.
\end{abstract}

Key words: Rhinella poeppigii, reproductive events, mating system, botanical garden.

Introducción.

Muchos anfibios exhiben ciclos de vida bifásicos, utilizando hábitats acuáticos para la reproducción y desarrollo larvario, y hábitats terrestres para el crecimiento adulto, forrajeo, hibernación y dispersión (Price et al., 2007). El uso de múltiples hábitats ha sugerido que sean propuestos como buenos indicadores de las condiciones ecológicas imperantes (Price et al., 2007).

Con respecto a los anuros, Kühnel et al., (2010), señalan que su actividad reproductiva es extremadamente diversa entre las especies y dentro de las mismas. Aquellas especies cuya estación reproductiva dura de 1 a 14 días son consideradas reproductoras explosivas, mientras que en aquellas en las que la temporada reproductiva se extiende de un mes a un año, se denominan reproductoras prolongadas. Cabe destacar que en estas últimas pueden alternarse períodos de intensa actividad vocal reproductiva con momentos de silencio reproductivo
(McCauley et al., 2000). Se han postulado muchas hipótesis para explicar la duración de los episodios reproductivos, pero las condiciones ambientales (presencia de lluvias, altas temperaturas) y la disponibilidad de lugares apropiados para la reproducción son considerados factores importantes (Segev et al., 2012).

Rhinella poeppigii (Tschudi, 1945) es un anuro que pertenece a la familia Bufonidae cuya área de distribución corresponde a la cuenca del Alto Amazonas del Perú y las áreas andinas de Bolivia, entre los 350 - 2000 msnm (Angulo et al., 2004).

Los objetivos del presente estudio fueron reportar la presencia de Rhinella poeppiggi en el campus de la Universidad Nacional Agraria La Molina (UNALM), localizado en la costa del Perú, describir su actividad reproductiva y señalar algunos factores que influirían en la misma. 
Materiales y métodos.

Área de Estudio.

El campus de la Universidad Nacional Agraria La Molina (UNALM), de aproximadamente 220 hectáreas, está localizado en la costa del Perú, en la ciudad de Lima, distrito de La Molina, a una altura de 244 msnm. Las instalaciones constan principalmente de aulas, laboratorios, edificios administrativos, zonas de cultivo, granjas de animales y un área dedicada al Jardín Botánico (Takano \& Castro, 2007). Si bien el campus está dentro del casco urbano, cuenta con grandes áreas arborizadas que muchas veces sirven de refugio para la fauna silvestre.

Las observaciones de la presencia y reproducción de $R$. poeppigii fueron efectuadas en el Jardín Botánico "Octavio Velarde Núñez" (12 04' 44" S, $76^{\circ}$ 56' 40" W), en la Universidad Nacional Agraria La Molina, Lima, Perú desde inicios del mes de abril hasta fines del mes de noviembre del año 2012. Según datos del Observatorio Meteorológico Alexander Von Humboldt localizado dentro del campus, el clima presenta temperaturas mínimas mensuales promedio de $16.2^{\circ} \mathrm{C}$ y máximas de $22.5^{\circ} \mathrm{C}$, una humedad relativa promedio anual de $81 \%$ y una precipitación total anual de $10 \mathrm{~mm}$. El Jardín Botánico cuenta con un área aproximada de 2 hectáreas y alberga tres estanques artificiales cercanos a la edificación “Julio Gaudron” y al vivero.

Metodología.

Desde abril hasta noviembre del 2012, cada dos días, de las 20 a las 22 horas, guiados por la actividad vocal de los machos de $R$. poeppigii presentes en los estanques, se registró lo siguiente: número de individuos, sexo y comportamiento (i.e., interacción social y amplexo). La especie fue identificada en base a claves y al análisis taxonómico siguiendo a Pramuk \& Kadivar (2003) y De la Riva (2002). Se colectó un individuo de $R$. poeppigii registrando las siguientes medidas externas con un vernier caliper electrónico de resolución $0.01 \mathrm{~mm}$ : longitud hocico cloaca, longitud de la cabeza, ancho de la cabeza, longitud de la tibia, longitud de la pata, ancho del ojo, diámetro del ojo, longitud de la parótida, ancho de la parótida, distancia interorbital, longitud ojo-narina, longitud narina rostrum, distancia entre narinas y longitud del tímpano.

Asimismo, mediante el análisis de factores abióticos y bióticos se describió el microhábitat elegido para la reproducción. Las variables para caracterizar el microhábitat se seleccionaron siguiendo a (Hartel et al., 2007 y Wells, 2007). Las variables abióticas usadas fueron: área del estanque $\left(\mathrm{m}^{2}\right)$, profundidad máxima del estanque $(\mathrm{cm})$, probabilidad de secado (bajo, medio y alto), $\mathrm{pH}$, y disponibilidad de oxígeno (mg/lt). Las variables bióticas consideradas fueron: vegetación dominante (dentro y alrededor del estanque) y presencia de depredadores (invertebrados y vertebrados).

\section{Resultados.}

Se confirmó la identificación de Rhinella poeppigii a partir de las características aportadas por Pramuk \& Kadivar (2003) y De la Riva (2002) y tomando en cuenta las vocalizaciones aportadas por Márquez et al. (2002). Se registró un total de 98 ejemplares, 92 machos y 6 hembras, y se colectó un individuo macho (CORBIDI 12507) que fue identificado por el Curador de la División de Herpetología del Centro de Ornitología y Biodiversidad (CORBIDI). La morfometría del ejemplar se presenta en la Tabla 1.

Tabla 1. Medidas morfométricas del ejemplar macho adulto de Rhinella poeppigii (CORBIDI 12507) colectado en el Jardín Botánico Octavio Velarde Núñez, UNALM.

\begin{tabular}{lc}
\hline Medida & $(\mathrm{mm})$ \\
\hline Longitud hocico - cloaca & 108.75 \\
Longitud de la cabeza & 37.11 \\
Ancho de la cabeza & 47.47 \\
Longitud de la tibia & 46.64 \\
Longitud de la pata & 43.8 \\
Ancho del ojo & 10.16 \\
Diámetro del ojo & 12.64 \\
Longitud de la parótida & 20.77 \\
Ancho de la parótida & 11.52 \\
Distancia interorbital & 14.23 \\
Longitud ojo - narina & 7.59 \\
Longitud Narina- rostrum & 1.46 \\
Distancia entre narinas & 9.00 \\
Tímpano & 6.55 \\
\hline
\end{tabular}

Eventos reproductivos.

En los meses evaluados hubo tres eventos reproductivos de corta duración, ocurridos aproximadamente del 17 al 21 de abril, del 17 al 20 de septiembre y del 14 al 17 de octubre del año 2012 (Tabla 2).

Tabla 2. Número de machos participantes de Rhinella poeppigii por cada evento reproductivo.

\begin{tabular}{ccc}
\hline $\begin{array}{c}\text { Evento } \\
\text { Reproductivo }\end{array}$ & Fechas & $\begin{array}{c}\text { Número de } \\
\text { machos } \\
\text { participantes }\end{array}$ \\
\hline Primero & $17 / 04 / 12$ & 9 \\
& $19 / 04 / 12$ & 21 \\
Segundo & $17 / 04 / 12$ & 5 \\
Tercero & $19 / 09 / 12$ & 10 \\
& $14 / 10 / 12$ & 23 \\
& $16 / 10 / 12$ & 9 \\
\hline
\end{tabular}

Todos los eventos reproductivos coincidieron con el riego por inundación que se practica en forma eventual en el Jardín Botánico y también con una temperatura ambiental muy elevada o con el inicio del aumento en la temperatura ambiental correspondiente 
a los meses más cálidos del año (Figura 1). En el día 19 de setiembre, se registró el mayor número de participantes en el evento reproductivo y fue justamente en esa fecha en que se observó la única oviposición.

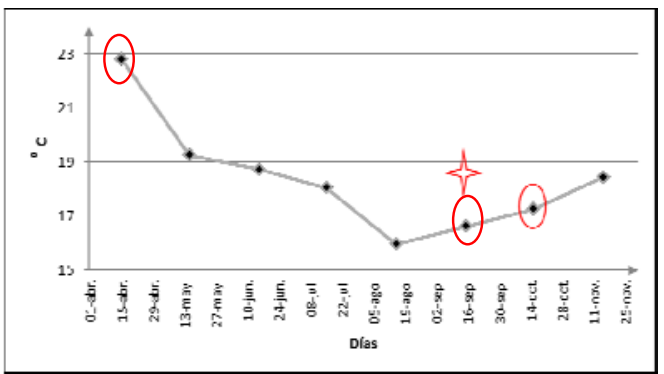

Figura 1. Temperatura ambiental media* en los meses evaluados en el Jardín Botánico Octavio Velarde Núñez. Los círculos indican la presencia de eventos reproductivos. La estrella señala la oviposición.

\section{Descripción de la actividad reproductiva.}

La mayor agregación de machos se registró el segundo día de los eventos reproductivos. La Figura 2 muestra ciertos comportamientos observados durante los eventos reproductivos. En los machos, se observó alternancia entre el llamado (Figura 2a) y la búsqueda. Se observó que los machos nadaban en búsqueda directa de hembras, registrándose varios intentos para acoplarse a ellas. Llamó la atención el hecho de registrar emparejamientos indiscriminados (macho con macho y hasta un intento de amplexo con una paloma mostrado en la Figura 2b). Durante el desarrollo de estas acciones fue que se tomó en cuenta la poca presencia de hembras (solo dos por evento). El amplexo fue del tipo axilar, no habiendo registro de competencia por las hembras ya acopladas. En dicho amplexo, el macho se monta sobre la hembra y la abraza con sus patas anteriores, logrando sujetarse en la zona axilar (Figura 2c). Aunque no se presenció el proceso mismo de la oviposición, éste se evidenció solo en el segundo evento con el registro de los largos cordones de huevos que fueron dejados entre la vegetación sub-acuática. El reporte del desarrollo de los huevos se reserva para otra publicación.

Estanques.

El Jardín Botánico de la UNALM cuenta con 3 estanques localizados en la parte central del mismo. La Tabla 3 detalla los factores abióticos y bióticos evaluados en cada uno de ellos.

Todos los eventos reproductivos se llevaron a cabo en el Estanque 1 (Figura 3). A partir de los datos presentados en la Tabla 3 , se observa que este

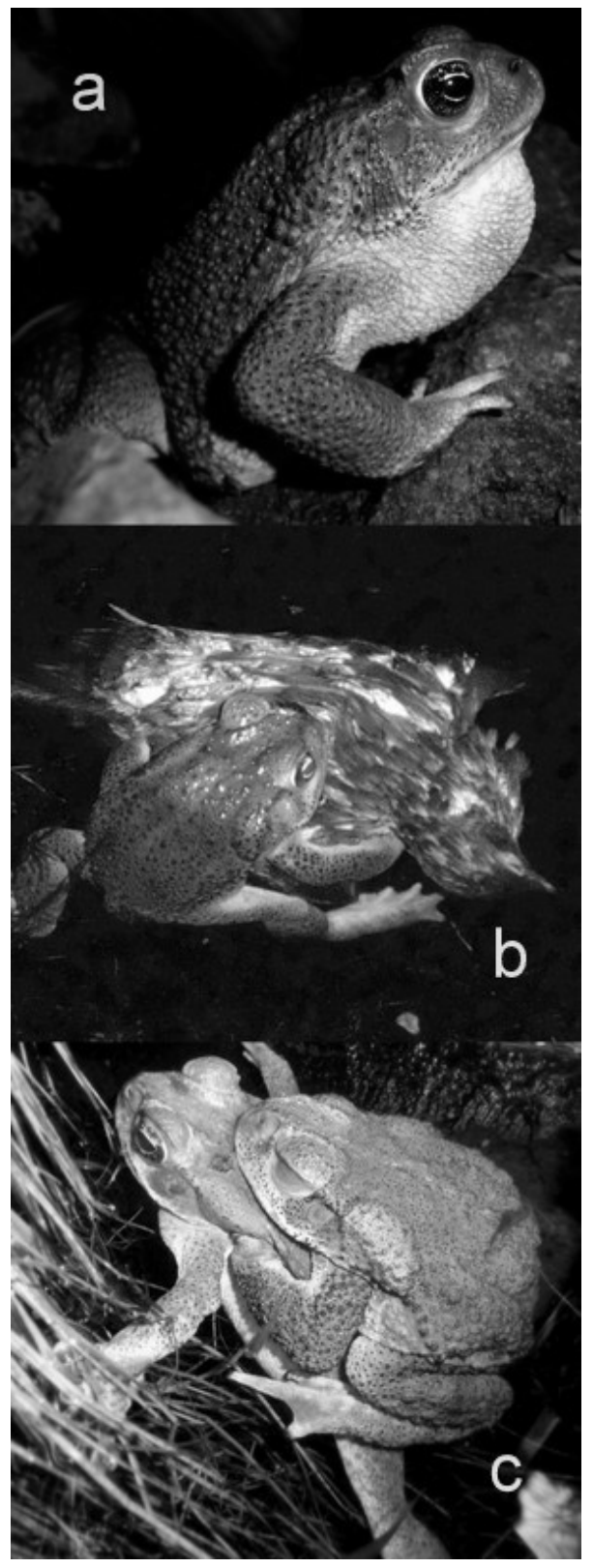

Figura 2. Algunos comportamientos observados durante los eventos reproductivos de Rhinella poeppigii.

$\mathbf{2 a}=$ macho cantando con saco vocal expandido,

$\mathbf{2 b}=$ sapo acoplado a paloma,

2c = pareja de $R$. poeppigii en amplexo.

estanque presenta mayor área, baja probabilidad de secado, pH más alcalino, y mayor disponibilidad de oxígeno. Elodea sp. y diversas especies de algas fueron la vegetación dominante, no encontrándose vertebrados como depredadores (p.ej. Oreochromiss sp.) pero sí la presencia de libélulas náyade. 


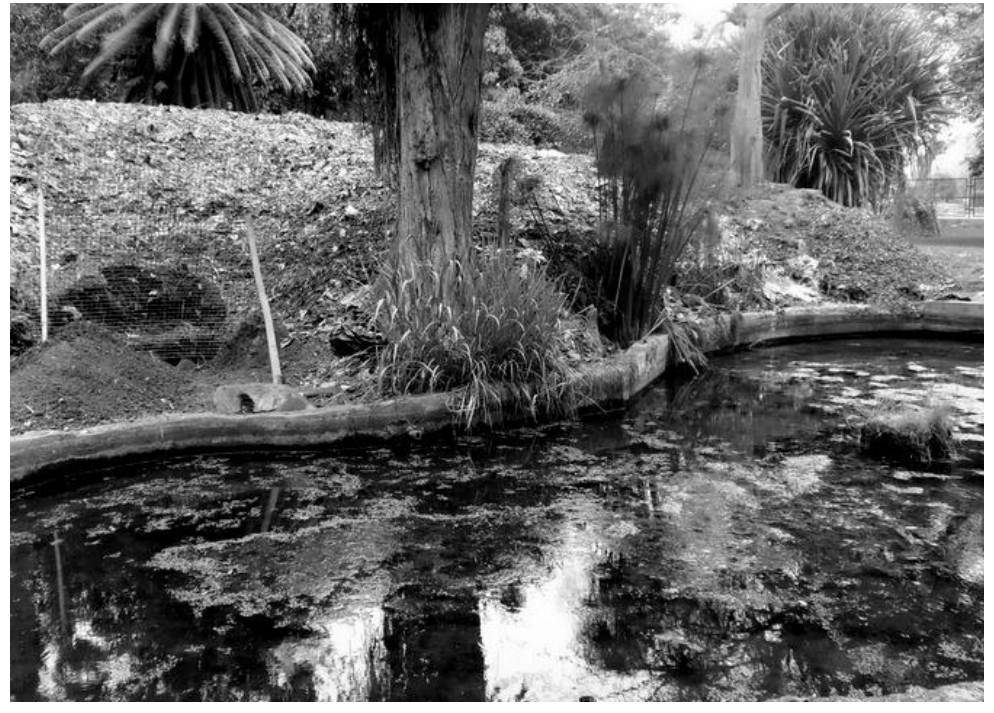

Figura 3. Estanque utilizado para la actividad reproductiva de Rhinella poeppigii. estribaciones orientales de los Andes en Perú y Bolivia. Según Frost (2009) esta especie se distribuye en los bosques húmedos montanos de los Andes de Ecuador, Perú y Bolivia, desde los 800 hasta los 1670 m.s.n.m. Sin embargo, en este estudio se registró no solamente su presencia en el desierto costero, en el Departamento de Lima, en un área antrópica (el Jardín Botánico de la Universidad Nacional Agraria La Molina), sino que también se pudo observar sus eventos reproductivos.

De acuerdo con Angulo et al. (2004) y Frost (2009) las especies del género Rhinella reportadas para el Departamento de Lima son $R$. limensis (Werner,

Tabla 3. Factores abióticos y bióticos en los 3 estanques del Jardín Botánico de la UNALM.

\begin{tabular}{|c|c|c|c|}
\hline \multirow[t]{2}{*}{ Variables } & \multicolumn{3}{|c|}{ Estanques } \\
\hline & 1 & 2 & 3 \\
\hline Área aprox. $\left(\mathrm{m}^{2}\right)$ & 39.7 & 34.2 & 5.4 \\
\hline Profundidad (cm) & 42 & 75 & 25 \\
\hline Probabilidad de secado & Baja & Baja & alta \\
\hline $\mathrm{pH}$ & 8.60 & 7.50 & 8.15 \\
\hline Disponibilidad de oxígeno (mg/l) & 6.90 & 2.85 & 6.25 \\
\hline Vegetación dominante dentro del estanque & $\begin{array}{l}\text { Elodea sp. y } \\
\quad \text { algas }\end{array}$ & Nymphaea sp. & $\begin{array}{l}\text { Elodea sp. y } \\
\text { algas }\end{array}$ \\
\hline Vertebrados depredadores & - & Oreochromis sp. & - \\
\hline Invertebrados depredadores & libélulas náyade & - & - \\
\hline Vegetación dominante alrededor del estanque & $\begin{array}{c}\text { Gramíneas, } \\
\text { Aracaceae, } \\
\text { Cyperus } \\
\text { papyrus, } \\
\text { Poaceaea, } \\
\text { Hojarasca } \\
\text { acumulada }\end{array}$ & $\begin{array}{c}\text { Cyperus papyrus, Canna } \\
\text { indica, Strelitzia nicoloai } \\
\text { Hidrocotyle bonariensis, } \\
\text { Justicia spicigera, } \\
\text { Gramíneas }\end{array}$ & $\begin{array}{c}\text { Limonium sp. } \\
\text { Paspalum sp., } \\
\text { Cupressus sp. } \\
\text { Lamiaceae, } \\
\text { Gramíneas }\end{array}$ \\
\hline
\end{tabular}

\section{Discusión.}

Tanto las medidas morfométricas de Rhinella poeppigii consignadas en la Tabla 1, como la comparación de características de las posibles especies presentes en el área de estudio consignadas en el Anexo 1, permiten afirmar que los ejemplares observados en los eventos reproductivos en el Jardín Botánico de la UNALM, corresponden a la especie $R$. poeppigii.

De acuerdo con Angulo et al., (2004) R. poeppigii se encuentra en los bosques montanos de las
1901) cuya distribución es a lo largo del desierto costero peruano desde Pisco (Ica) hasta el norte de Sechura (Piura), desde los 70 hasta los 2830 metros de elevación y $R$. spinulosa (Wiegmann, 1834), cuya distribución también se sobrepone, pero sobre los 1000 metros de altitud (Aguilar et al. 2005). Debido a la disyuntiva encontrada, puesto que según el análisis Pramuk \& Kadivar (2003) y De la Riva (2002) los individuos colectados pertenecerían a $R$. poeppigii, se efectuó una comparación de medidas entre $R$. poeppigii y $R$. limensis (Anexo 1 ) utilizando ejemplares de la la Colección del Centro de Ornitología y Biodiversidad (CORBIDI). Una de las 
mayores diferencias encontradas es la longitud de la parótida, que en $R$. limensis está alrededor de $9 \mathrm{~mm}$, alcanzando un tamaño mediano, mientras que en $R$. poeppigii asciende a unos $20 \mathrm{~mm}$, es decir un tamaño considerablemente mayor. Cabe destacar que en $R$. poeppigii, las parótidas son subtriangulares (De la Riva, 2002) y los individuos observados en el Jardín Botánico tenían las parótidas de esa forma.

Por otro lado, tomando en cuenta las características de la piel, se conoce que $R$. limensis puede tener formaciones lenticulares o hemisféricas con proyecciones córneas múltiples, mientras que siempre se presentan en $R$. spinulosa. Además, cuando $R$. limensis las presenta, son abundantes lateralmente y en $R$. spinulosa son abundantes tanto lateral como dorsalmente (Aguilar et al., 2005). La piel de $R$. poeppigii es siempre rugosa, con tubérculos de tamaño similar, recubiertos por espículas queratinizadas. La piel de los machos es usualmente marrón sin ningún patrón, mientras que la de las hembras tiene parches pequeños más oscuros no muy bien definidos (De la Riva, 2002).

Resulta fuera del alcance de esta publicación analizar los factores que expliquen la presencia de $R$. poeppigii fuera de su ámbito de distribución sino más bien de documentar tanto su presencia como su reproducción en el desierto costero. Según las observaciones de campo (vocalizaciones y comportamiento), las medidas obtenidas y la información bibliográfica, puede afirmarse que $\mathrm{R}$. poeppigii está presente en la costa, y que se está reproduciendo en un ambiente antrópico de la ciudad de Lima.

De la Riva (2002) menciona que los machos de $R$. poeppigii, durante la época reproductiva, se congregan alrededor de pozas efímeras y charcos tanto en el lecho de ríos aislados como dentro de poblados. Nuestros resultados apoyan esta última aseveración, de allí que no resulte sorprendente haber podido observar la existencia de eventos reproductivos en un estanque dentro de un Jardín Botánico.

Bionda et al., (2012) señalan que la urbanización es la responsable de la disminución de las poblaciones de anfibios a nivel mundial, pero Rubbo \& Kiesecker (2005) indican que hay especies frecuentemente halladas en lugares urbanos o suburbanos que son más resilientes al desarrollo urbano. Por ejemplo, Segev et al. (2012) documentan que Dyscophus antongili, una especie de la costa este de Madagascar, se reproduce todo el año en un área urbana y la perturbación antropogénica, no tiene consecuencias negativas para su conservación. Rubbo \& Kiesecker (2005) postulan que esas especies tendrían ciertas características de su historia natural que las hacen menos vulnerables a la urbanización. Ese sería el caso de $R$. poeppigii, que no solamente está presente en una zona fuera de su ámbito de distribución, sino que además se han registrado eventos reproductivos.
De acuerdo con Rodrigues \& Cerqueira (2010), la mayor parte de los anuros tropicales restringen su actividad reproductiva a los períodos de lluvia. En la ciudad de Lima, la lluvia se limita a pequeños episodios de garúa durante los meses de invierno y muy escasos chubascos durante el verano, no siendo comparable esta situación con la estacionalidad de las lluvias en los ecosistemas amazónicos. Los eventos reproductivos de Rhinella poeppigii habrían sido desencadenados por el sistema de riego eventual practicado en el Jardín Botánico, aunque éstos no hayan sido necesariamente determinantes de dicha actividad. Duellman (1992), citado por CáceresAndrade \& Urbina-Cardona (2009), remarca que la lluvia no siempre está garantizada para los anuros de las llanuras, por lo que deben enfrentar el reto de encontrar la humedad adecuada para el desarrollo tanto de los estadios larvarios como de los adultos.

Dado que los eventos reproductivos son de restringida duración, con agregación de muchos machos, abundantes vocalizaciones y emparejamientos indiscriminados, se estaría evidenciando que la reproducción en $R$. poeppigii es de tipo explosivo (Wells, 1997). Los emparejamientos indiscriminados se asemejan a lo encontrado por Roberto et al. (2011) para Rhinella hoogmoedi y a lo mencionado por Wells (1997), quien señala que muchos reproductores explosivos exhiben emparejamientos indiscriminados. La escasa presencia de hembras también ha sido documentada por Penna et al. (2005) quienes reportan que en las áreas reproductivas las hembras son escasamente vistas. La diversidad de estrategias en inversión energética en los huevos explicaría la única oviposición que se presenció en los tres eventos (Reading, 1991; Pough et al., 1992). Posteriormente se observó el desarrollo de estas larvas, y dichos resultados se reservan para otra publicación.

Según Rubbo \& Kiesecker (2005), la respuesta de cada especie al ambiente depende de las complejas interacciones entre los factores locales y bióticos. La elección de la especie del Estanque 1 para la reproducción podría deberse a ciertas condiciones facilitadas por éste tales como mayor disponibilidad de oxígeno, baja probabilidad de secado y relativo mayor $\mathrm{pH}$, las cuales podrían tener una influencia positiva en el crecimiento y metamorfosis de los renacuajos (Sadinski \& Dunson, 1992; Wells, 2007). Con respecto a los factores bióticos, se estaría postulando que la vegetación sub-acuática del estanque permitiría la adhesión de los huevos y reduciría la posibilidad de que éstos sean desecados (Wells, 2007). La ausencia de Oreochromis sp. en dicho estanque puede haber sido determinante en la elección, ya que varios cíclidos se consideran potenciales depredadores de los huevos y larvas en anfibios (Wells, 2007) y se sabe que algunos sapos tienden evitar la oviposición en estanques con la 
presencia de peces predadores (Holomuzki, 1995). Existe información de que en ecosistemas amazónicos dentro de las charcas temporales sin peces, las náyades de libélulas son los principales depredadores de huevos y larvas de anfibios pero según Wells (2007) la presencia de náyades de libélula, no tendría tanto impacto comparado con el de peces predadores.

\section{Conclusiones.}

Se reporta por primera vez la presencia de Rhinella poeppigii en la costa del Perú, en un área antrópica del departamento de Lima (campus de la UNALM). Los tres eventos reproductivos observados en el Jardín Botánico fueron de corta duración, con poca presencia de hembras y hasta con un acoplamiento con una paloma. Los factores que estarían desencadenando la reproducción de esta especie, serían el sistema de riego practicado en el Jardín Botánico y los aumentos en la temperatura ambiental. Se registró la selección de un microhábitat específico para la reproducción (Estanque 1) con alta disponibilidad de oxígeno, baja probabilidad de secado, con presencia de algas como vegetación dominante dentro del estanque y con la existencia de libélulas náyade como depredadoras. En esencia, lo que se ha podido documentar en este trabajo es que, al igual que para muchos anuros, la presencia y reproducción de $R$. poeppigii vendría a estar determinada por la compleja interacción entre sus requerimientos fisiológicos y las características de su hábitat particular.

\section{Agradecimientos.}

Al profesor Aldo Ceroni por el permiso para el trabajo en el Jardín Botánico, a la profesora Claudia Caro por sus recomendaciones iniciales y a la profesora Mercedes Flores por la ayuda en el reconocimiento en las especies botánicas. A Alfredo Beraún, Ana Lucía Mares, Manuel Quispe, Daniel Tasayco, Vicky Berrocal, Julio Salvador, Jorge Luján, Piero Calderón y a todos los demás voluntarios por su colaboración en el trabajo de campo. Finalmente se le agradece a Pablo Venegas, Director de la División de Herpetología en CORBIDI, por el apoyo a la presente investigación.

\section{Literatura citada.}

Aguilar C., Ramírez C., Rivera D., Siu-Ting K., Suárez J. \& Torres C. 2005. Anfibios andinos del Perú fuera de áreas naturales protegidas: amenazas y estado de conservación. Rev. peru. Biol. 17 (1): 5-28.

Angulo A., De la Riva I. \& Jungfer K.H. 2004. Rhinella poeppigii. En: IUCN 2012. IUCN Red List of Threatened Species. Version 2012.2. $<$ www.iucnredlist.org > . Downloaded on 25 December 2012.

Bionda C., Salas N., Caraffa E., Baraquet M. \& Martino A. 2012. On abnormalities recorded in an urban population of Rhinella arenarum from central Argentina. Herpetology Notes. 5: 237-241.
Cáceres-Andrade, S. \& Urbina-Cardona J. N. 2009. Ensamblajes de anuros de sistemas productivos y bosques en el piedemonte llanero, Departamento del Meta, Colombia. Caldasia. 31 (1):175-194.

De la Riva I. 2002. Taxonomy and distribution of the South American toads, Bufo poeppigii Tschudi, 1845 (Amphibia, Anura, Bufonidae). Graellsia: 49-57.

Duellman W. E. \& Trueb L. 1986. Biology of amphibians. Páginas 13 - 47. New York: McGraw-Hill.

Frost D.R. 2009. database. Amphibian Species of the World: an Online Reference v5.3.

2013. Amphibian Species of the World: an Online Reference. Version 5.6 (9 January 2013). Base de datos electrónica disponible en http://research.amnh.org/herpetology/amphibia/index.ht ml. American Museum of Natural History, New York, USA.

Hartel, T., Nemes, S., Mara, G. 2007. Breeding phenology and spatio-temporal dynamics of pond uses by the yellow-bellied toad (Bombina variegata) population: the importance of pond availability and duration. Acta Zoologica Lituana. 17: 57-63.

Holomuzki J. 1995. Oviposition sites and fish-deterrent mechanisms of two stream anurans. Copeia 1995:60713.

Kühnel S., Reinhard S. \& Kupfer A. 2010. Evolutionary reproductive morphology of amphibians: an overview. Bonn Zoological Bulletin. 57: 119-126.

Márquez, R., De la Riva I., Bosch J. \& Matheu E. Editores.2002. Guía Sonora de las Ranas y Sapos de Bolivia. Publicado por Alosa, sonidos de la naturaleza y Fonoteca Zoologica. Disponible en http://www.fonozoo.com/eng/versonidoAmphibia.php?i $\mathrm{d}=592359$ \& registro $=p u$

McCauley S. J., Bouchard S. S., Farina B. J., Isvaran K., Quader S., Wood D. W. \& St. Mary C. M. 2000. Energetic dynamics and anuran breeding phenology: Insights from a dynamic game. Behav. Ecol. 11:429-36.

Penna M., Narins P. \& Feng A. 2005. Thresholds for evoked vocal responses of Eupsophus emiliopugini (Amphibia, Leptodactylidae).Herpetologica. 61(1): 1-8.

Pramuk J.B. \& Kadivar F. 2003. A New Species of Bufo (Anura: Bufonidae) from Southern Ecuador. Herpetologica. 59(2):270-283.

Price S. J., Howe R. W., Hanowski J. M., Regal R. R., Niemi G.J. \& Smith C. R. 2007. Are Anurans of Great Lakes Coastal Wetlands Reliable Indicators of Ecological Condition?. J. Great Lakes Res. 33 (Special Issue 3): 211-223.

Pough F. H., Magnusson W. E., Ryan M. J., Wells K. D. \& Taigen T. L. 1992. Behavioral energetics. In Environmental physiology of the amphibians, ed. M. E. Feder and W. W. Burggren, 395-436. Chicago: University of Chicago Press.

Reading C.J. 1991. The relationship between body length, age and sexual maturity in the common toad, Bufo bufo. Holarctic Ecology. 14: 245-249.

Roberto I.J., Brito L. \& Cascon P. 2011. Temporal and spatial patterns of reproductive activity in Rhinella hoogmoedi (Anura: Bufonidae) from a tropical rainforest in northeastern Brazil, with the description of it's advertisement call. South American Journal of Herpetology. 6(2): 87-97. 
Rodrigues da Silva F. \& de Cerqueira Rossa-Feres D. 2010. Seasonal variation in body size of tropical anuran amphibians. Herpetology Notes. 3: 205-209.

Rubbo M. J. \& Kiesecker J. M. 2005. Amphibian Breeding Distribution in an Urbanized Landscape. Conservation Biology. 19 (2): 504-511.

Sadinski W.J. \& Dunson W.A. 1992. A multilevel study of effects of low $\mathrm{pH}$ on amphibians of temporary ponds. J. Herpetol. 26, 413-422.

Segev, O., Andreone F., Pala R., Tessa G. \& Vinces M. 2012. Reproductive phenology of the tomato frog,
Dyscophys antogili, in an urban pond of Madagascar's east coast. Acta Herpetologica. 7 (2): 331-340

Takano F. \& Castro N. 2007. Avifauna en el campus de la Universidad Nacional Agraria La Molina (UNALM), Lima - Perú. Ecología Aplicada. 6(1, 2): 149 - 154.

Wells K.D. 1977. The social behaviour of anuran amphibians. Animal Behaviour 25, 666-693.

2007. The ecology \& behavior of amphibians. University of Chicago Press. Chicago.

Anexo 1. Comparación de las medidas de dos individuos adultos de Rhinella limensis (CORBIDI 10735) y Rhinella poeppigii (CORBIDI 12507)

\begin{tabular}{ccc}
\hline Medidas (mm) & R. limensis & R. poeppigii \\
\hline Longitud hocico - cloaca & 106.69 & 108.75 \\
Longitud de la cabeza (del margen & 36.48 & 37.11 \\
posterior de la mandíbula inferior hasta & & \\
la punta del hocico) & & 47.47 \\
Ancho de la cabeza & 43.47 & 10.16 \\
Ancho del párpado superior & 8.65 & 12.64 \\
Diámetro del ojo (medido & 10.5 & 14.23 \\
horizontalmente) & & \\
Distancia interorbital(entre los & 12.12 & 9.00 \\
párpados superiores) & & 20.77 \\
Distancia entre narinas & 7.28 & 11.52 \\
Longitud de parótida & 9.79 & 46.64 \\
Ancho de parótida & 8.06 & 43.8 \\
Longitud de Tibia & 43.04 & 7.59 \\
Longitud de pata & 42.52 & 1.46 \\
Longitud ojo-narina & 7.21 & 6.55 \\
Longitud narina- rostrum & 1.14 & \\
Longitud tímpano & 4.00 & \\
\hline
\end{tabular}

1 Departamento de Biología. Facultad de Ciencias. Universidad Nacional Agraria La Molina, Lima Perú. Correspondencia: Mwilliams@lamolina.edu.pe

${ }^{2}$ Estudiante de Biología. Facultad de Ciencias. Universidad Nacional Agraria La Molina. 